\title{
Exposure of $38 \mathrm{~nm}$ period grating patterns with extreme ultraviolet interferometric lithography
}

\author{
H. H. Solak, D. He, W. Li, S. Singh-Gasson, and F. Cerrina ${ }^{a)}$ \\ Department of Electrical and Computer Engineering, University of Wisconsin-Madison, Madison, \\ Wisconsin 53706 \\ B. H. Sohn, X. M. Yang, and P. Nealey \\ Department of Chemical Engineering, University of Wisconsin-Madison, Madison, Wisconsin 53706
}

(Received 12 November 1998; accepted for publication 18 August 1999)

\begin{abstract}
Extreme ultraviolet (EUV, $\lambda=13 \mathrm{~nm}$ ) lithography is considered to be the most likely technology to follow ultraviolet (optical) lithography. One of the challenging aspects is the development of suitable resist materials and processes. This development requires the ability to produce high-resolution patterns. Until now, this ability has been severely limited by the lack of sources and imaging systems. We report printing of $38 \mathrm{~nm}$ period grating patterns by interferometric lithography technique with EUV light. A Lloyd's Mirror interferometer was used, reflecting part of an incident beam with a mirror at grazing incidence and letting it interfere with the direct beam at the wafer plane. High-density fringes (38 nm pitch) were easily produced. Monochromatized light of $13 \mathrm{~nm}$ wavelength from an undulator in an electron storage ring provided the necessary temporal and spatial coherence along with sufficient intensity flux. This simple technique can be extended to sub-10 nm resolution. (C) 1999 American Institute of Physics. [S0003-6951(99)02841-7]
\end{abstract}

Extreme ultraviolet lithography (EUVL, $\lambda=13.4 \mathrm{~nm}$ ) is one of the candidate technologies pursued for printing semiconductor devices with critical dimensions of $70 \mathrm{~nm}$ and below. ${ }^{1}$ The approaches currently developed are based on the use of $4 \times$ reduction optics and reflective masks. The reflectivity of near-normal incidence optics is enhanced to $\sim 60 \%-$ $70 \%$ by multilayer interference coatings ( $\lambda / 4$ stacks). In this spectral region, all materials have high absorption coefficients bringing some special requirements on EUVL optical systems and materials. For example, photoresist thickness has to be limited to less than $\sim 0.1 \mu \mathrm{m}$ in order to obtain a sufficiently uniform exposure along the film thickness.

Evaluation of resist materials require the ability to expose patterns in order to obtain measurements of exposure dose sensitivity, contrast and resolution using a given material. A prototype EUV projection camera has been built at the Sandia National Laboratory that is capable of printing sub- $0.1 \mu \mathrm{m}$ lines and spaces but access is limited. ${ }^{2}$

We have developed a system using EUV-interferometric lithography (EUV-IL) for studying imaging at EUV wavelengths, and for testing photoresist materials in which we have demonstrated unprecedented printing of features with periods as small as $38 \mathrm{~nm}$. IL was not used before to print such small features in the EUV regime, and the features are the smallest ever printed using a photon-based lithography. Fabrication of quantum confinement devices is a clear application, and another is the study of the properties of materials confined in ultrasmall structures (polymeric resists in particular).

IL is commonly used for production of large area gratings and grids ${ }^{3}$ and possible extension into imaging twodimensional features is studied. ${ }^{4}$ In principle, it allows the

${ }^{a}$ Corresponding author. Electronic mail: cerrina@nanotech.wisc.edu production of fine features over large areas without the need for complicated masks and imaging systems. The depth of focus of an interferometric system is practically unlimited, affording great flexibility in sample positioning. We note that IL is widely used in the ultraviolet and some attempts have been made in vacuum ultraviolet (VUV) and harder $\mathrm{x}$ rays, with limited success. ${ }^{5-7}$ An early experiment in $\mathrm{VUV}^{5}$ demonstrated the potential of interference techniques although the wavelength used $(118 \mathrm{~nm})$ was too large to print features in the 10-20 nm regime. Tatchyn et al. ${ }^{6}$ used an undulator source with a transmission grating as a monochromator in a Lloyd mirror scheme. Their results were limited by temporal coherence and available flux. Interference between different diffraction orders from a transmission grating were used by Wei et al. ${ }^{7}$ to print fringe patterns. The fringe period in that case was determined by the grating period and in practice vibration and drift problems limited the quality of the printed pattern.

The fringe period $d$ created by two plane waves intersecting at an angle $\left(\theta_{1}+\theta_{2}\right)$ as seen in Fig. 1(a), is given by $\lambda /\left(\sin \theta_{1}+\sin \theta_{2}\right)$. This sets an ultimate limit of $6.7 \mathrm{~nm}$ for minimum printable feature period at $\lambda=13.4 \mathrm{~nm}$. The target feature size for EUV lithography is roughly in the $20-100$ $\mathrm{nm}$ range requiring a shallow intersection angle in the $2^{\circ}-$ $10^{\circ}$ range. A Lloyd's Mirror interferometer used in conjunction with a synchrotron radiation source offers an easy implementation and was in fact employed in some preliminary work. ${ }^{6}$ More recently, the technique was successfully used for synchrotron source characterization. ${ }^{8}$ In a Lloyd's Mirror interferometer schematically shown in Fig. 1(a), the light from a point source $S_{1}$ is incident on a mirror at a grazing angle. The reflected beam interferes with the direct beam and creates a fringe pattern on the screen $A A^{\prime}$. If the angle of incidence on the mirror is small then $\theta_{1} \simeq \theta_{2} \simeq \theta$ and 


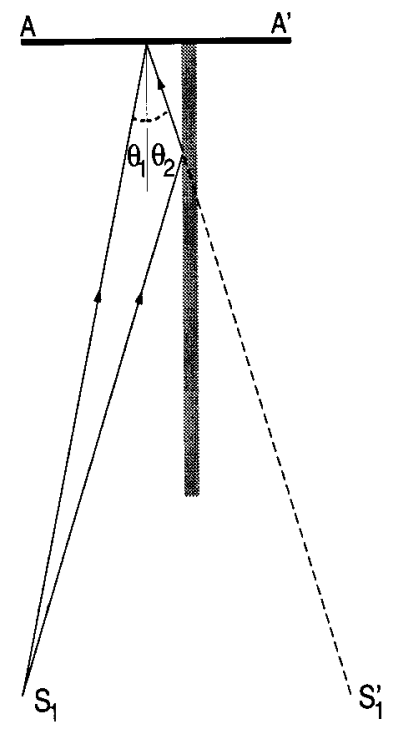

(a)

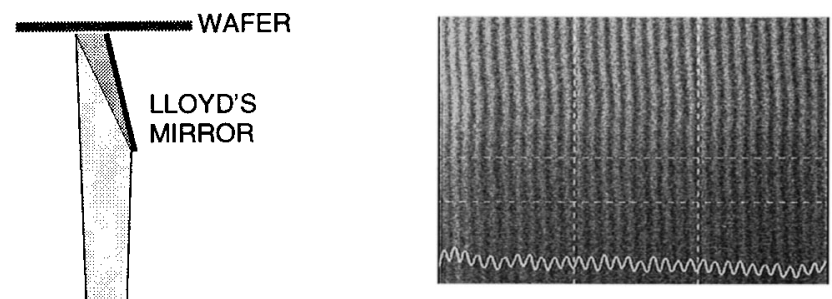

(a)

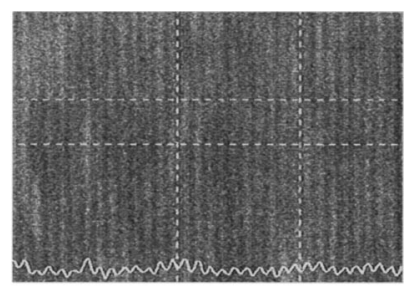

(b)

FIG. 2. SEM images of EUV interferograms on (a) 100 nm PMMA, (b) 100 nm UV6. Both patterns have $38 \mathrm{~nm}$ pitch.

seems to have better performance at this extreme resolution although fringe patterns are clearly resolved on both films. The nonuniformity in those images is believed to be due to the speckle pattern created by the surface roughness of the Au mirror. It is difficult to obtain high quality images of such thin photoresist films with SEM due to low contrast and charging related problems even though the SEM had adequate resolution. Likewise, the wiggles in the fringe patterns is due to the beam induced charging in the SEM. Atomic force microscope (AFM) images in Fig. 3 of two other interferograms demonstrate a better imaging solution for these patterns. The silicon nitride AFM tips used in this experiment have a $70^{\circ}$ opening angle. Thus, they can penetrate only about $13 \mathrm{~nm}$ into the $19 \mathrm{~nm}$ (half-pitch) wide trenches. The origin of the apparent roughness in these patterns is currently under study.

The number of fringes observable in the Lloyd Interferometer depends on the temporal and spatial coherence properties of the source. The finite temporal coherence limits the allowed optical path difference between the direct and reflected beams for obtaining visible fringes. Therefore, the number of fringes $m$ is limited by

$$
m \ll \frac{\lambda}{\Delta \lambda} .
$$

Experimentally, the slits of the monochromator were set to obtain a $\lambda / \Delta \lambda \simeq 1000$. Therefore, for a $0.04 \mu \mathrm{m}$ fringe period, we can expect to see fringes in a $1000 \times 0.04=40 \mu \mathrm{m}$ range starting from the edge of the Au mirror.

The spatial coherence of the light comes into play in conjunction with the source size, i.e., the exit slit of the monochromator in our experimental setup. The length of the

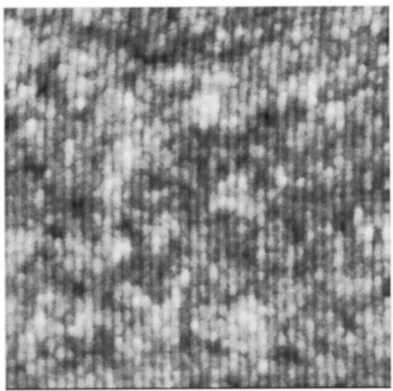

(a)

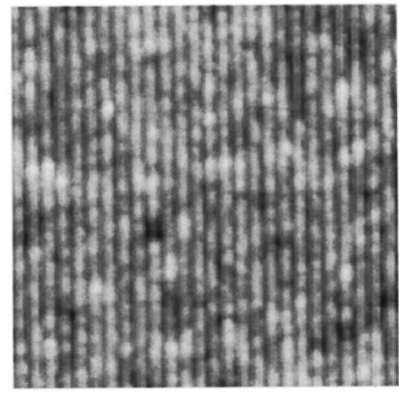

(b)

micrographs of $38 \mathrm{~nm}$ period fringe patterns printed on 100 $\mathrm{nm}$ thick polymethyl methacrrylate (PMMA) and UV6 photoresist film from Shipley Inc. ${ }^{10}$ The areas shown were near the edge of the mirror within a $\sim 10 \mu \mathrm{m}$ distance. PMMA the edge of the mirror within a $\sim 10 \mu \mathrm{m}$ distance. PMMA on PMMA.

FIG. 3. AFM images of EUV interferograms. (a) $3.7 \times 3.7 \mu \mathrm{m}$ image of 50 $\mathrm{nm}$ pitch pattern on UV6 resist. (b) $1 \times 1 \mu \mathrm{m}$ image of $38 \mathrm{~nm}$ pitch pattern

to AIP license or copyright, see http://apl.aip.org/apl/copyright.jsp 
slits in the horizontal direction [perpendicular to the plane of the Fig. 1(b)] has no effect on the fringe pattern to a first approximation since moving a point source in that direction would only move the fringes along their length. ${ }^{11}$ The "width" (w) however is critical. For an ideally incoherent illumination of the slit, the visibility of the fringes exhibit an oscillatory decrease from the mirror side with a $1 / x$ type envelope. For a $10 \mu \mathrm{m}$ exit slit width used in our experiments, the visibility goes below 0.5 for distances more than $500 \mu \mathrm{m}$ away from the mirror. ${ }^{12}$ This should allow recording of more than 12000 fringes assuming a $40 \mathrm{~nm}$ fringe period. Hence, in our experimental setup, temporal coherence ultimately limits the number of fringes and the source can be considered to be spatially coherent for the practical purpose. Thus, the Lloyd's mirror interferometer makes perfect use of the source, requiring coherence only in one direction, i.e., along the slit width. It is interesting to note that coherence properties of EUV and x-ray light from modern synchrotron sources is increasingly being exploited in studies such as $\mathrm{x}$-ray holography, coherent x-ray diffraction and interferometry; which are characterization and measurement techniques in nature. EUV interferometry in particular is used for at wavelength characterization of EUVL optics. ${ }^{13,14}$ Our use of coherent synchrotron light for production of nanostructures represents an expansion of this exploitation in an new direction.

In conclusion, we have demonstrated that it is possible to use interferometric lithography in the EUV for printing grating patterns with periods as small as $38 \mathrm{~nm}$. Lloyd's mirror is a simple and elegant scheme that takes advantage of the good coherence properties of monochromatized undulator radiation. We notice that EUV-IL can be extended to gratings with periods as small as $6 \mathrm{~nm}$ for the development of new nanostructures. Other advantages offered by EUV radiation (beside the short wavelength) are very small photoelectron blur due to relatively low photon energy and ability to use thin resists due to high absorption coefficients ( $\mu$ $\simeq 5 \mu \mathrm{m}^{-1}$ ). Work is under way for extension of the technique to even smaller features and variable frequency fringe patterning.
The authors thank Michael Green for the help with the calculations related to the properties of the undulator source. This work is based in part by an Intel Research Customization grant from the Semiconductor Research Corporation, No. 97-LJ-481. The Center for X-ray Lithography is supported in part by DARPA/ONR Grant No. N00014-97-10460. The Synchrotron Radiation Center is operated under NSF Award No. DMR-95-310009.

${ }^{1}$ For recent developments in the field see Emerging Lithographic Technologies II, edited by Y. Vladimirsky [Proc. SPIE 3331, (1998)].

${ }^{2}$ J. E. M. Goldsmith, P. K. Barr, K. W. Berger, L. J. Bernardez, G. F. Cardinale, J. R. Darnold, D. R. Folk, S. J. Haney, C. C. Henderson, K. L. Jefferson, K. D. Krenz, G. D. Kubiak, R. P. Nissen, D. J. O'Connell, Y. E. Perras, A. K. Ray-Chaudhuri, T. G. Smith, R. H. Stulen, D. A. Tichenor, A. A. Ver Berkmoes, and J. B. Wronosky, Proc. SPIE 3331, 11 (1998).

${ }^{3}$ T. A. Savas, M. L. Schattenburh, J. M. Carter, and H. I. Smith, J. Vac. Sci. Technol. B 14, 4167 (1996).

${ }^{4}$ S. H. Zaidi and S. R. J. Brueck, J. Vac. Sci. Technol. B 11, 658 (1993).

${ }^{5}$ G. C. Bjorklund, S. E. Harris, and J. F. Young, Appl. Phys. Lett. 25, 451 (1974).

${ }^{6}$ R. Tatchyn, E. Kallne, A. Toor, T. Cremer, and P. Csonka, Rev. Sci. Instrum. 60, 1579 (1989).

${ }^{7}$ M. Wei, D. T. Attwood, and T. K. Gustafson, J. Vac. Sci. Technol. B 12, 3648 (1994).

${ }^{8}$ O. V. Chubar, Proceedings of the 1995 Particle Accelerator Conference on IEEE, 1996, p. 2447.

${ }^{9}$ M. C. Hettrick and J. H. Underwood, Appl. Opt. 25, 4228 (1986).

${ }^{10}$ Shipley Inc., 455 Forest St., Marlboro, MA 01752.

${ }^{11}$ M. Born and E. Wolf, Principles of Optics, 4th ed. (Pergamon, London, 1970).

${ }^{12}$ This problem is solved in Ref. 11 for the case of Fresnel's Mirrors. Similar treatment yields the following result for fringe visibility in the case of Lloyd's mirror for an incoherent source:

$$
V=\frac{\left|\sin \frac{\pi w x}{l \lambda}\right|}{\frac{\pi w x}{l \lambda}},
$$

where $w$ is the source width, $x$ is the distance of the observation point from the mirror, $l$ is the distance between the source and the observation plane, and $\lambda$ has the usual meaning. Note that the fringe visibility is independent of the angle of incidence on the mirror, i.e., the fringe period.

${ }^{13}$ D. T. Attwood, K. Halbach, and K.-J. Kim, Science 228, 1265 (1985).

${ }^{14}$ E. Tejnil, K. A. Goldberg, S. H. Lee, H. Medecki, P. J. Batson, P. E. Denham, A. A. MacDowell, J. Bokor, and D. Atwood, J. Vac. Sci. Technol. B 15, 2455 (1997). 